\title{
Association of infantile bruxism and the terminal relationships of the primary second molars
}

\author{
Tatiana Helena Junqueira(a) \\ Ana Carla Raphaelli Nahás- \\ Scocate ${ }^{(a)}$ \\ Karyna Martins do Valle-Corotti(a) \\ Ana Claudia de Castro Ferreira \\ Conti ${ }^{(b)}$ \\ Shirley Trevisan ${ }^{(c)}$

\footnotetext{
(a) Department of Orthodontics, College of Dentistry, Univ Cidade de São Paulo UNICID, São Paulo, SP, Brazil.

(b) Department of Orthodontics, College of Dentistry, Univ Norte do Paraná, Londrina, PR, Brazil.

(c) College of Dentistry, Univ Cidade de São Paulo - UNICID, São Paulo, SP, Brazil.
}

Declaration of Interests: The authors certify that they have no commercial or associative interest that represents a conflict of interest in connection with the manuscript.

\section{Corresponding Author:}

Ana Carla Raphaelli Nahás-Scocate

E-mail: carlanahas@yahoo.com.br

Submitted: Jul 06, 2012

Accepted for publication: Sep 17, 2012

Last revision: Oct 05, 2012

\begin{abstract}
The aim of this study was to analyze the association between infantile bruxism and the terminal relationships of the primary second molars. A total of 937 pre-school children (both genders), aged from 2 to 6 years, from municipal schools in São Paulo were evaluated. In this study, a questionnaire considering the bruxism habit and the presence of headaches and/or restless sleep was answered by the parents/guardians. A clinical exam of occlusion in the anteroposterior direction (vertical plane - VP, mesial step - MS and distal step - DS) was performed by the examiners in the school environment. Student's $t$ test, Fisher's test and a logistic regression test were applied for the statistical analysis at a significance level of $5 \%$. The prevalence of the bruxism habit was $29.3 \%$ among the total sample. Because there was no significant difference between the sides evaluated, the left side was taken as the standard. Among those children with bruxism, $25.7 \%$ presented a mesial step terminal relationship at the primary second molars, $29.1 \%$ had DS, and $30.2 \%$ had VP. Regarding the association of the parafunctional habit with the type of terminal relationship, no significant results were found. Children who slept restlessly or suffered from headaches were verified to show a higher chance of expressing the habit ( $\mathrm{OR}=2.4$ and 1.6 , respectively). The prevalence of bruxism in the studied sample was $29.3 \%$, and its association with the primary second molars' terminal relationship was not statistically significant.
\end{abstract}

Descriptors: Malocclusion; Dentition; Bruxism; Epidemiology.

\section{Introduction}

Bruxism is a parafunctional activity of the masticatory system that is characterized by "tooth-clenching or grinding", with rhythmic muscular contractions, being more frequent during sleep. ${ }^{1,2}$ A certain controversy exists in the literature regarding the etiological factors of this parafunctional habit in childhood. It has been characterized as a multifactorial habit comprising hereditary, psychological and behavioral factors; the environment; and occlusal and some pathological interferences..$^{2-5}$ During primary dentition, a thorough clinical exam must be carefully performed, not only to diagnose caries but also to assess occlusion and the masticatory and cervical musculature, which could confirm or exclude a diagnosis of the presence or absence of a deleterious functional habit.

There is a shortage of studies in the literature that evaluate the preva- 
lence of bruxism in children, making it difficult to establish the scientific parameters and its association with etiological factors. The majority of existing studies do not report the association between this habit and occlusal factors, such as overjet, overbite, openbite and crossbite, ${ }^{3,6}$ that affect the relationship between the molars and canines. In addition, no study in the literature relates bruxism in the primary dentition considering the primary second molar terminal relationship. Childhood bruxism has been diagnosed in children with temporomandibular disorders and in those with frequent headaches and nocturnal restlessness. ${ }^{7,8}$

The main aim of this investigation was to analyze the association between infantile bruxism and the primary second molars terminal relationships. In addition, the association of this parafunctional habit with headache and restless sleep was verified.

\section{Methodology}

This study was developed according to established precepts by the National Research Ethics Committee, approved under the number 13355774. This cross-sectional epidemiological study was conducted in children, ranging from 2 years and one month to 6 years and eleven months of age, at the stage of complete primary dentition, enrolled at 6 nursery schools in the eastern area of São Paulo city, SP, Brazil. The initial study began with 1011 children, with 74 not returning. Therefore, the final sample consisted of 937 children (479 boys and 458 girls). Subjects of mixed African/European descent predominanted $(57.6 \%)$, followed by those of European descent $(35.0 \%)$, African descent $(6.8 \%)$ and Asian descent $(0.5 \%)$. The following inclusion criteria were considered:

- a parent's or guardian's consent,

- absence of extensive carious lesions that would interfere with the patient's bite, dental losses or anomalies,

- absence of a history of traumatism, visual, auditory and/or mental deficiencies and

- absence of previous orthodontic and speech therapy treatments.

All of these factors would undermine the results of the study.

A questionnaire comprising standardized questions regarding a comprehensive history of the patient was answered by the parents/guardians. Based on the questionnaire, the presence of the parafunctional habit of bruxism, the period in which the child presented the habit, and the variables of the presence of restless sleep and headaches could be investigated. It is worth mentioning that there was no interference by the examiners while the questionnaires were being filled out.

The clinical examination was performed by three previously trained orthodontists, and the Kappa concordance index, $\kappa:$ 0.89-1.00, and Pearson's coefficient of correlation, Rs $>0.9$, confirmed a high inter-examiner reliability. The orthodontists performed the extraoral palpation and the intraoral direct visual inspection that enabled them to assess findings such as the occlusal relationships in the maximum habitual intercuspation (MHI). To classify the relationship between the primary second molars' distal surfaces, the criteria proposed by Baume were applied: ${ }^{9,10}$

1. vertical plane (VP) - the distal surfaces of the primary second molars coincide in the same vertical plane;

2. mesial step (MS) - the distal surface of the mandibular primary second molar is located in a more mesial position relative to the distal surface of the maxillary primary second molar; and

3. distal step (DS) - the distal surface of the mandibular primary second molar is located in a more distal position relative to the distal surface of the maxillary primary second molar.

Statistical evaluations were performed using Stata 8.0 software (StataCorp. LP, College Station, USA). Fisher's test was applied at the significance level of $5 \%$ to verify possible associations among the studied variables. In addition to measuring the magnitude of these associations, the chances of developing the parafunctional habit of bruxism were also evaluated (OR: odds ratio calculation).

\section{Results}

Among the total sample, the prevalence of brux- 
ism in children was $29.3 \%$, with $27.3 \%$ presenting the habit during the night and $2.0 \%$ during the day. When considering the terminal planes, MS was present in $17.3 \%$ of the sample, DS in $10.5 \%$ and VP in $72.3 \%$. These characteristics were evaluated for both sides of the dental arches, and the prevalence of VP was $72.1 \%$ for the right side (RS) and $72.2 \%$ for the left side (LS). For MS, 15.4\% and 17.3\% were present on the left and right sides, respectively, and for DS, $12.5 \%$ and $10.5 \%$ were present for the RS and LS, respectively.

To assess whether there was an association between the characteristic of the RS and that of the LS, Fisher's exact test was applied, and the p-value found was lower than 0.001. Children who presented VP, (90.2\%), MS (72.2\%) and DS (73.5\%) on the left side also presented the same characteristics on the right side. Therefore, the left side was considered to be standard for the association with the other evaluated characteristics. We observed that $31.5 \%$ of children who ground their teeth presented VP, $22.2 \%$ presented MS and $26.5 \%$ presented DS. When the bruxism habit was associated with sleep disorders, of the total number of children who pre- sented restless sleep (34.9\%), 38.8\% presented bruxism during the night, and $4 \%$ during the day (Figure 1). Regarding the presence of headaches associated with bruxism, of the children who had headaches during the day $(14.4 \%), 35.6 \%$ presented nocturnal bruxism, and $4.4 \%$ presented daytime bruxism. Among the children who had nocturnal headaches (2.9\%), $48.1 \%$ presented the parafunctional habit at night. It is worth emphasizing that $4.4 \%$ of the children evaluated presented headaches occasionally and $78.3 \%$ did not (Figure 2).

According to Fisher's statistical test (Table 1), only the variables of restless sleep and headaches were verified to show significant results when associated with the parafunctional habit of bruxism.

Table 1 - Fisher's Exact Test - Bruxism x Variables.

\begin{tabular}{c|c}
\hline Comparisons & P value \\
\hline Right molar & .595 \\
\hline Left molar & .052 \\
\hline Restless sleep & $.000^{*}$ \\
\hline Headache & $.003^{*}$ \\
\hline
\end{tabular}

$* P<.05$
Table 2 - Logistic regression model for the association of the parafunctional habit of bruxism with the significant co-variables studied.

\begin{tabular}{|c|c|c|c|c|c|c|c|c|}
\hline \multirow{3}{*}{\multicolumn{2}{|c|}{ Characteristic }} & \multicolumn{4}{|c|}{ Teeth grinding } & \multirow{4}{*}{$P$ value } & \multirow{4}{*}{ OR } & \multirow{3}{*}{$\mathrm{Cl}(95 \%)$} \\
\hline & & \multicolumn{2}{|c|}{ Yes } & \multicolumn{2}{|c|}{ no } & & & \\
\hline & & $\mathrm{n}$ & $\%$ & $\mathrm{n}$ & $\%$ & & & \\
\hline \multirow{2}{*}{ Restless sleep } & yes & 140 & 50.9 & 187 & 28.2 & & & \\
\hline & no & 135 & 49.1 & 475 & 71.8 & $.000^{*}$ & 2.4 & $1.8 ; 3.3$ \\
\hline \multirow{2}{*}{ Headache } & yes & 83 & 30.2 & 122 & 18.4 & & & \\
\hline & no & 192 & 69.8 & 540 & 81.6 & $.006^{*}$ & 1.6 & $1.1 ; 2.2$ \\
\hline
\end{tabular}

$P<.05$.

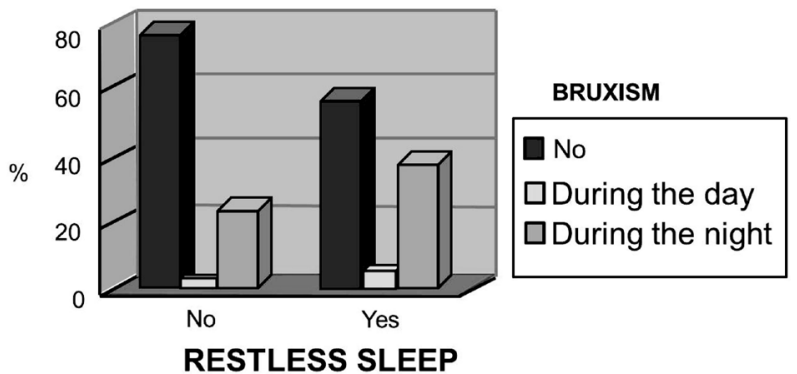

Figure 1 - Distribution of the total sample in percentages (\%) for the relationship between restless sleep and the parafunctional habit of bruxism.

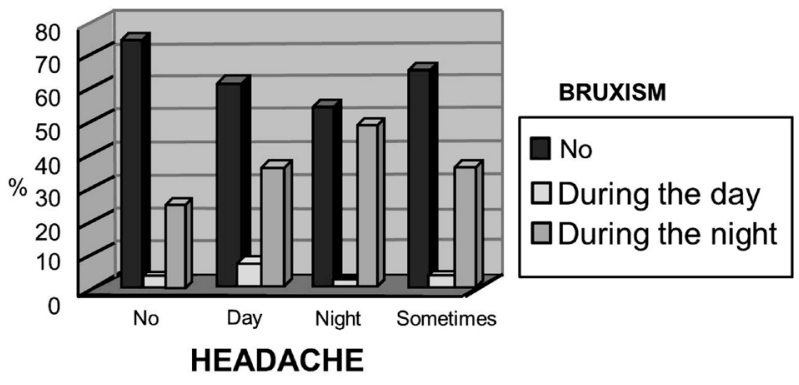

Figure 2 - Distribution of the total sample in percentages (\%) for the relationship between headaches and the parafunctional habit of bruxism. 
In the logistic regression model (Table 2), for the purpose of analyzing the possible associations between the significant co-variables and bruxism, the OR in the adjusted model for the restless sleep characteristic was 2.4 , i.e., children with restless sleep showed a 2.4-fold higher chance of presenting bruxism compared with children who did not present this characteristic. Regarding headaches, children who suffered from headaches presented a 1.6-fold higher chance of having the tooth grinding habit compared with those children who did not present headaches.

\section{Discussion}

Given that the possible etiological factors (social, systemic, hereditary, psychological, local and physiological factors) associated with childhood bruxism are still uncertain, ${ }^{11,12}$ this paper is of great importance to the literature. Some authors believe that this parafunctional activity of the masticatory system has a multifactorial etiology. ${ }^{3,4,13,14}$ In addition to the relevant aspect of this study design being based on a substantial sample size, which involved 937 children in the primary dentition stage, several characteristics were analyzed. This study comprised the occlusal characteristics in the antero-posterior direction of the dental arches, the presence of the parafunctional habit of bruxism, restless sleep and headaches.

The applied methodology, which involved a questionnaire answered by parents and/or guardians, to diagnose childhood bruxism, followed a similar standard to that used in other studies. ${ }^{4,5,8,15-17}$ The difficulty in diagnosing this parafunctional disturbance, mainly based on polysomnography exams, which was not performed in this sample, is known, ${ }^{2}$ and it becomes even more limited when one is dealing with children at the stage of primary dentition.

The results obtained in this research showed a high prevalence of the parafunctional habit of childhood bruxism (29.3\%), supporting further investigation on this topic. ${ }^{1,3,16-19}$ The literature has demonstrated a significant frequency of this habit compared with temporomandibular dysfunctions, which are less prevalent in this age group. ${ }^{12}$ However, there are studies that have found lower frequen- cies, ranging from $5.9 \%{ }^{1,20,21}$ to $11 \%{ }^{8,15}$ The origin of these differences regarding childhood bruxism is assumed to potentially be related to the lack of methodological uniformity in the studies, cultural differences existent among countries, ${ }^{21,22}$ or even by the variety in the age groups studied. ${ }^{5,23}$ Those features that may be considered normal in one age group may not be the case in another age group.

When evaluating the association of the presence of bruxism with the terminal relationships of the primary second molars, no significant relationship was found. These data could not be correlated with those of other studies because no similar studies associating these two aspects within the studied age group were found. Therefore, the local factor of $o c$ clusion is suggested to not be related to the bruxism habit at the stage of primary dentition, in agreement with the studies of Vanderas and Manetas ${ }^{6}$ and Demir et al., ${ }^{3}$ although these studies did not assess only primary dentition.

The variable of headache was significantly associated with the parafunctional habit of bruxism $(P=.003 *)$. Additionally, children who suffered from headaches presented a 1.6-fold higher chance of presenting the parafunctional habit of bruxism compared with children without this complaint. In a study of 90 children, Vendrame et al. ${ }^{8}$ related several types of headache with sleep disturbances. Headaches caused by stress represented $50 \%$ of the patients with the parafunctional habit of bruxism, and these patients had twice the chance of somaticizing the habit compared with children with other types of headache. Therefore, there is evidence that the emotional factor greatly influences the establishment of the bruxism habit, which is emphasized in several studies. ${ }^{11,15,18,19}$

Another important variable studied was restless sleep, with a prevalence of $34.9 \%$ among the total sample. When bruxism was associated with restless sleep, it showed the highest degree of significance $(P=.000 *)$. Children with a history of restless sleep present a 2.4-fold higher chance of expressing the parafunctional habit of bruxism. The results of this research suggest that there is a direct relationship between the bruxism habit and headaches and restless sleep, showing evidence of a relationship 
between emotional factors and the etiology of this habit. The presence of emotional stress ${ }^{5,24}$ was affirmed to show a greater influence on bruxism compared with other factors, ${ }^{25}$ including occlusal factors. It is worth emphasizing that a simple occlusal correction is not indicated as therapy for bruxism as instituting coadjutant therapies is necessary. However, stating that the emotional factor is the single triggering factor of bruxism would be incorrect. The combination of several etiological factors present in a single individual increases the probability of childhood bruxism appearing. Antônio, Pierro and Maia ${ }^{11}$ affirmed that the high prevalence of the parafunctional habit of bruxism found in recent studies is being considered an intrinsic problem of modern societies and has become a common condition in the child population.

There is no single method to definitely eliminate bruxism, but rather a group of therapeutic

\section{References}

1. Ghanizedeh A. ADHD, bruxism and psychiatric disorders: does bruxism increase the chance of a comorbid psychiatric disorder in children with ADHD and their parents?. Sleep Breath. 2008 Nov;12(4):375-80.

2. Hoz-Aizpurua JL, Díaz-Alonso E, LaTouche-Arbizu R, MesaJiménez J. Sleep bruxism. Conceptual review and update. Med Oral Patol Oral Cir Bucal. 2011 Mar;16(2):e231-8.

3. Demir A, Uysal T, Guray E, Basciftci FA. The relationsship between bruxism and occlusal factors among seven- to 19- yearold Turkish children. Angle Orthod. 2004 Oct;74(5):672-6.

4. Liu X, Ma Y, Wang Y, Jiang Q, Rao X, Lu X, et al. Brief report: an epidemiologic survey of the prevalence of sleep disorders among children 2 to 12 years old in Beijing, China. Pediatrics. 2005 Jan;115(1 Suppl):266-8.

5. Serra-Negra JM, Ramos-Jorge ML, Flores-Mendoza CE, Paiva SM, Pordeus IA. Influence of psychosocial factors on the development of sleep bruxism among children. Int J Paediatr Dent. 2009 Sep;19(5):163-9.

6. Vanderas AP, Manetas KJ. Relationship between malocclusion and bruxism in children and adolescents: a review. Pediatr Dent. 1995 Jan-Feb;17(1):7-12.

7. Ângeles ET, Gáldos AC, Sanches LR, Martinez CS. Prevalencia de bruxismo en pacientes de primeira vez en el Instituto Nacional de Pediatría. Acta Pediatr Mex. 2003 Mar-Abr;24(2):95-9.

8. Vendrame M, Kaleyias J, Valencia I, Legido A, Kothare SV. Polysomnographic findings in children with headaches. Pediatr Neurol. 2008 Jul;39(1):6-11. measures $^{1}$ is used because the etiology of bruxism is varied. The therapeutic approach to sleep bruxism at present is oriented toward palliating, albeit partially, the effect of sleep bruxism and preventing and treating its pathological effect on the stomatognathic apparatus. ${ }^{2}$ The great influence of emotional factors ${ }^{26}$ must not be forgotten, and the dentist must always opt for a non-invasive multidisciplinary treatment that involves pediatricians, psychologists, pediatric dentists and otorhinolaryngologists.

\section{Conclusions}

The prevalence of the parafunctional habit of bruxism was $29.3 \%$ among the total sample studied. There was no significant relationship between this parafunctional habit and the terminal relationships of the primary second molars. However, headache and restless sleep were significantly associated with infantile bruxism.

9. Baume LJ. Physiological tooth migration and its significance for the development of occlusion. I. The biogenetic course of the deciduous dentition. J Dent Res. 1950 Apr;29(2):123-32.

10. Baume LJ. Physiological tooth migration and its significance for the development of occlusion. II. The biogenesis of accessional dentition. J Dent Res. 1950 Jun;29(3):331-7.

11. Antonio AG, Pierro VSS, Maia LC. Bruxism in children: a warning sign for psychological problems. J Can Dent Assoc. 2006 Mar;72(2):155-9.

12. Barbosa TS, Miyakoba LS, Pocztaruk RL, Rocha CP, Gavião MBD. Temporomandibular disorder and bruxism in childhood and adolescence: review of the literature. Int J Pediatr Otorhinolaryngol. 2008 Mar;72(3):299-314.

13. Rios D, Magalhães AC, Honório HM, Buzalaf MA, Lauris JRP, Machado MAAM. The prevalence of deciduous tooth wear in six-year-old children and its relationship with potential explanatory factors. Oral Health Prev Dent. 2007 Aug;5(3):167-71.

14. Vélez AL, Restrepo CC, Vargas AP, Gallego GJ, Alvarez E, Tamayo V, et al. Head posture and dental wear evaluation of bruxist children with primary teeth. J Oral Rehabil. 2007 Sep;34(9):663-70.

15. Bharti B, Malhi P, Kashyap S. Patterns and problems of sleep in school going children. Indian Pediatr. 2006 Jan;43(1):35-8.

16. Villa MT, Torres AM, Soto BB, Gomar MR, Langa MJS, Sierra YAIU. Patrones normales y tratornos del sueño entre la población escolar de la ciudad de Gandía. An Pediatr (Barc). 2008 Jan;68(3):250-6. 
17. Serra-Negra JM, Paiva SM, Seabra AP, Dorella C, Lemos BF, Pordeus IA. Prevalence of sleep bruxism in a group of brazilian schoolchildren. Eur Arch Paediatr Dent. 2010 Aug;11(4):1925.

18. Ng Dk, Kwok K, Cheung JM, Leung S, Chan CH. Prevalence of sleep problems in Hong Kong primary school children. Chest. 2005 Sep;128(3):1315-23.

19. Petit D, Touchette E, Tremblay RE, Boivin M, Montplaisiur J. Dyssomnias and parasomnias in early childhood. Pediatrics. 2007 May;119(5):1016-25.

20. Agargun MY, Clli AS, Sener SS, Bilici M, Ozer OA, Selvi Y, et al. The prevalence of parasomnias in preadolescent Schoolaged Children; a Turkish sample. Sleep. 2004 Feb;27(4):701-5.

21. Lam MH, Zhang J, Li AM, Wing YK. A community study of sleep bruxism in Hong Kong children: association with comorbid sleep disorders and neurobehavioral consequences. Sleep Med. 2011 Aug;12(7):641-5.
22. Liu X, Liu L, Owens JA, Kaplan LD. Sleep patterns and sleep problems among schoolchildren in the United States and China. Pediatrics. 2005 Jan;115(1):241-9.

23. Pérez RL, Morales PL, Yañez AB, Maupomé G, Vídrio PG. Prevalence of bruxism among Mexican children whit Down syndrome. Down Syndr Res Pract. 2007 Jul;12(1):45-9.

24. Vanderas AP, Menenakou M, Kouimtzis TH, Papagiannoulis L. Urinary catecholamine levels and bruxism in children. J Oral Rehabil. 1999 Feb;26(2):103-10.

25. Laberge L, Tremblay RE, Vitaro F, Montplaisir J. Development of parasomnias from childhood to erly adolescence. Pediatrics. 2000 Jul;106(1):67-74.

26. Restrepo CC, Alvarez E, Jaramillo C, Vélez C, Valencia I. Effects of psychological techniques on bruxism in children with primary teeth. J Oral Rehabil. 2001 Apr;28(4):354-60. 